\title{
El aprendizaje basado en problemas: una propuesta de cualificación docente
}

Autor de correspondencia: ${ }^{1}$ rubinsten.hernandez@uptc.edu.co Recibido: 06 de junio de 2020 Revisado: 30 julio de 2020 Aprobado: 10 de diciembre de 2020 Publicado: 06 de marzo de 2021

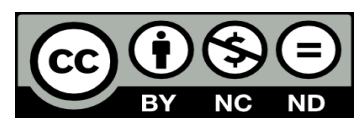

Rubinsten Hernández Barbosa ${ }^{1}{ }^{(0)}$, Sandra Maritza Moreno Cardozo Universidad Pedagógica y Tecnológica de Colombia, Gimnasio Moderno, Colombia

\section{Resumen}

En este texto se describe una experiencia investigativa de cualificación con profesores de ciencias naturales, que tuvo por objetivo que ellos se apropiaran de una estrategia de trabajo escolar y así consideraran alternativas que favorecieran el desarrollo de competencias, de pensamiento crítico y de construcción de conocimiento científico escolar; además de que reconocieran el valor del trabajo en equipo para favorecer el desarrollo de aprendizajes. La propuesta recoge la experiencia, las consideraciones y las sugerencias de dos años de implementación. Se desarrolló a partir de una estructura organizada en etapas y se tuvo presente la coherencia entre los elementos conceptuales de la estrategia de aprendizaje basado en problemas y su puesta en marcha. La estrategia toma como base la literatura existente en el campo, pero se ajustó a los contextos específicos de desarrollo. Los resultados permitieron evidenciar la potencia de la estrategia. Se destaca el trabajo cooperativo, los conceptos estructurantes y el proceso metacognitivo, entre otros aspectos; pero, a la vez, se reconocen sus limitaciones en relación con el tiempo de diseño del problema y la puesta en marcha de la estrategia.

Palabras clave: aprendizaje basado en problemas, estrategia didáctica, pensamiento científico, pensamiento crítico, trabajo en equipo 


\title{
Problem-based learning: a proposal for teacher qualification
}

\begin{abstract}
This text describes a research experience of qualification with natural science teachers, which had the objective of them adopting a strategy of educational work and thus considering alternatives that would favour the development of competences, of critical thinking, and of school scientific knowledge construction; as well as recognizing the value of team work to favour the learning development. The proposal reflects the experience, considerations and suggestions gained during two years of implementation. It was developed based on a staged structure and the coherence between the conceptual elements of the problem-based learning strategy and its implementation was considered.. The strategy is based on existing documentation in the area but adjusted to the specific context of development. The results revealed the potential of the strategy. The cooperative work, the structuring concepts, and the metacognitive process, among other aspects, are highlighted; but, at the same time, their limitations are recognized regarding the time required for the design of the problem and the implementation of the strategy.
\end{abstract}

Keywords: problem-based learning, teaching strategy, scientific thinking, critical thinking, teamwork

\section{Aprendizagem baseada em problemas: uma proposta de qualificação docente}

\section{Resumo}

Este texto descreve uma experiência de investigação de qualificação com professores de ciências naturais, cujo objetivo foi que eles se apropriassem de uma estratégia de trabalho escolar e, assim, considerassem alternativas que favorecessem o desenvolvimento de competências, o pensamento crítico e a construção do conhecimento científico escolar; além de reconhecer o valor do trabalho em equipe para promover o desenvolvimento da aprendizagem. A proposta inclui a experiência, considerações e sugestões de dois anos de implementação. Foi desenvolvida a partir de uma estrutura organizada em etapas e foi considerada a coerência entre os elementos conceituais da estratégia de aprendizagem baseada em problemas e sua implementação. A estratégia baseia-se na literatura existente no campo, mas foi ajustada a contextos específicos de desenvolvimento. Os resultados mostraram o potencial da estratégia. $\mathrm{O}$ trabalho cooperativo, os conceitos estruturantes e o processo metacognitivo, entre outros aspectos, são destacados; mas, ao mesmo tempo, suas limitações são reconhecidas em relação ao tempo de desenho do problema e a implementação da estratégia.

Palavras-chave: aprendizagem baseada em problemas, estratégia didática, pensamento científico, pensamento crítico, trabalho em equipe 
En los espacios de las clases de ciencias, los maestros se encuentran con preguntas de los estudiantes a propósito del sentido y la pertinencia de lo que se aprende. Es común escuchar en sus voces: “¿Esto para qué me sirve? ¿Eso ya lo vimos el año pasado? ¿Toca hacer laboratorio?". Todas estas preguntas nos llevan a reflexionar sobre las representaciones limitadas que han construido sobre la ciencia escolar, en donde se privilegian aprendizajes declarativos de conceptos científicos, que de manera repetitiva se desarrollan en cada grado escolar y con una visión de ciencia ajena, donde las verdades son absolutas y acabadas. Esto reduce, entonces, la potencia del pensamiento científico y la aleja de una tesis fundamental: la ciencia como una construcción social y cultural, que responde a necesidades específicas en espacios sociales, políticos, económicos y geográficos determinados. Las representaciones de los estudiantes están marcadas por las experiencias que se construyen en las aulas, donde las estrategias pedagógicas, en ocasiones, terminan siendo repetitivas y los procesos de transposición didáctica no logran desarrollar el espíritu investigativo ni el pensamiento científico. Frente a esta problemática, los autores desarrollaron una investigación en donde proponen, a partir de la formación docente, implementar una estrategia didáctica para aplicar con los estudiantes, fundamentada en el aprendizaje basado en problemas [ABP]. Con ello, se pretende el desarrollo del pensamiento científico a partir de problemas situados y contextuales y la integración disciplinar que promueva la formación de ciudadanos críticos y propositivos. Pero esto no puede ocurrir sin que se movilicen las concepciones docentes y se abran espacios de discusión y reflexión para repensar y reconfigurar las acciones docentes en el aula, lo cual fue el propósito de esta investigación.

El ABP es una estrategia didáctica que favorece el aprendizaje por indagación. Moviliza y potencia el desarrollo del pensamiento científico y crítico, el trabajo en equipo y la autonomía, entre otros aspectos. Para su diseño e implementación es necesario considerar la formación tanto de docentes como de los estudiantes. Para el primer caso, se requiere de un modelo de fundamentación, donde los aspectos teóricos y prácticos se vayan entretejiendo para reconfigurar nuevas formas de pensar la enseñanza y el aprendizaje de un ámbito del conocimiento como STEM. Es precisamente bajo esta premisa que los autores diseñaron y desarrollaron un modelo de intervención con el propósito de cualificar docentes en formación y en ejercicio, el cual se sustenta en el ABP. A continuación, se plantean algunos elementos de orden teórico que sirvieron como principios orientadores epistemológicos y didácticos para desarrollar la propuesta de trabajo que se describe en este texto.

El ABP tiene su origen en la década de los 60, en las universidades de McMaster —en Canadá- y Case Western Reserve - en Estados Unidos-, en programas de ciencias de la salud y, de manera particular, en medicina (Cohen-Schotanus et al., 2008; Prince et al., 2005). Como punto articulador contó con las metodologías activas (Rhem, 1998). Su génesis en la formación médica estuvo enmarcada por la pretensión y la exigencia de cambiar el desarrollo curricular de contenidos basados en temas por uno fundamentado en problemas de la vida real, que permitiera la integración y convergencia de diferentes áreas de conocimiento, con el fin de plantear soluciones a problemas reales a los que se debe enfrentar un futuro médico.

El ABP se fundamenta epistemológicamente en el constructivismo de perspectiva social. En esta teoría de aprendizaje, 
el conocimiento no es el resultado de una mera copia de la realidad preexistente, sino que es un proceso dinámico e interactivo a través del cual la información externa es interpretada por la mente que va construyendo progresivamente modelos cada vez más complejos y potentes. (Coll, 1998, p. 38)

A continuación, se caracterizan algunos aspectos esenciales a la hora de poner en marcha la estrategia didáctica por $\mathrm{ABP}$ :

- El aprendizaje significativo requiere de una actividad mental, que permite al sujeto establecer diferentes tipos de relaciones entre el conocimiento nuevo y los contenidos que subyacen en su estructura cognoscitiva.

- El aprendizaje se da en la interacción entre el docente, los estudiantes, los recursos y el contenido mismo que es objeto de estudio y análisis, lo cual genera nuevos significados y esquemas mentales de conocimiento.

- Los aprendizajes significativos son más potentes cuando son construidos a partir de situaciones cotidianas o de situaciones que se pueden aplicar en soluciones de problemas contextuales. Dependen tanto del nivel del desarrollo cognitivo como del nivel de competencia del estudiante. Por ello, es fundamental reconocer el papel mediador del docente, así como las actividades y tareas que él propone para que se potencie la zona de desarrollo próximo (Vygotski, 1993).

- Los intereses, motivaciones, actitudes, experiencias y expectativas que tienen los estudiantes son determinantes en la construcción del conocimiento.

- Los conocimientos previos permiten establecer vínculos, andamiajes y relaciones con los nuevos, los cuales deben orientarse de manera no arbitraria.

- La colaboración del docente para que el estudiante construya una voz propia, desde el análisis del problema hasta su resolución, es fundamental, pues posibilita construir una imagen de sujeto que piensa y plantea soluciones oportunas, al usar el conocimiento que construye desde sus potencialidades particulares.

La implementación del ABP, en cualquiera de sus niveles educativos y áreas de conocimiento, además del deseo y formación conceptual rigurosa por parte de quienes lo proponen (Oakley et al., 2007), requiere de dedicación y esfuerzo - tanto de los docentes como de los propios estudiantes-, que, sumados a la constancia y procesos de seguimiento y evaluación continuos, garantizan cierto nivel de éxito. También sus limitaciones e inconvenientes han sido documentados. Uno de ellos está relacionado con las dificultades del trabajo en grupo (Andreu-Andrés \& García-Casas, 2006).

El ABP busca aprendizajes situados y contextualizados. Para ello, la participación de los sujetos en la construcción de escenarios dialógicos e interactivos favorece la construcción de comunidades de práctica y aprendizaje, desde donde se reconstruyen nuevas formas de ser, pensar, actuar y comunicar, tanto en estudiantes como en docentes. Se visibiliza entonces la necesidad de poner en diálogo los saberes disciplinares de los docentes, que, por lo general, planean desde sus disciplinas y en solitario, sin que en las instituciones escolares se abran espacios para favorecer un currículo integrado en donde diferentes disciplinas entren en diálogo para construir experiencias a manera de vasos comunicantes y se consoliden conocimientos holísticos e integrados que permitan entender y dar solución a problemas de la realidad fracturada - tanto de ámbitos locales como nacionales e internacionales_-, y 
así proponer una planeación conjunta de manera creativa, en donde se construyan nuevas articulaciones desde los campos del saber y desde las voces y saberes de los maestros, para lograr otros vínculos con la historia que hoy nos habla.

\section{Metodología}

El enfoque de la experiencia investigativa se construyó desde el paradigma cualitativo, con un enfoque crítico - en tanto se pretende no solo comprender la realidad, sino transformarla- y con un diseño de estudio de caso. La investigación se desarrolló en el lapso de dos años, con dos grupos poblacionales: estudiantes y docentes en ejercicio. En el primer grupo, se trabajó con 130 estudiantes, con edades que oscilan entre 20 y 24 años, de Licenciatura en Química de la Universidad Distrital, específicamente en una asignatura denominada Resolución de Problemas, que en el programa corresponde al VII semestre. El segundo grupo corresponde a 20 docentes del Gimnasio Moderno que forman parte del ámbito STEM, con edades entre 30 y 50 años. En la tabla 1 se señalan las diez etapas que se llevaron a cabo para el desarrollo de la investigación. Las tres primeras fueron desarrolladas previamente por los autores; las demás, en conjunto con los docentes en formación y en ejercicio.

\section{Tabla 1}

Etapas de desarrollo de ABP con docentes en formación y en ejercicio

\begin{tabular}{|c|c|}
\hline Etapa & Objetivo \\
\hline $\begin{array}{l}\text { 1. Revisión sistemática de la } \\
\text { literatura. }\end{array}$ & $\begin{array}{l}\text { Localizar y sistematizar, en diferentes bases de datos, las fuentes } \\
\text { bibliográficas sobre ABP. }\end{array}$ \\
\hline $\begin{array}{l}\text { 2. Características del ABP según la } \\
\text { literatura revisada. }\end{array}$ & $\begin{array}{l}\text { Establecer y organizar, a partir de la literatura, las características } \\
\text { del ABP. }\end{array}$ \\
\hline $\begin{array}{l}\text { 3. Planeación y diseño de la } \\
\text { estrategia con los docentes en } \\
\text { formación y en ejercicio. }\end{array}$ & $\begin{array}{l}\text { Diseñar el modelo de trabajo con los profesores en formación y en } \\
\text { ejercicio. }\end{array}$ \\
\hline 4. Sensibilización & Motivar a los profesores a considerar el ABP como una estrategia. \\
\hline 5. Fundamentación teórica. & $\begin{array}{l}\text { Desarrollar los ejes conceptuales de la propuesta e internalizarlos a } \\
\text { partir del diseño y la reflexión sistemática. }\end{array}$ \\
\hline 6. Concepto y diseño del problema. & $\begin{array}{l}\text { Identificar y caracterizar los elementos que es necesario tener } \\
\text { presentes en el diseño del problema. }\end{array}$ \\
\hline $\begin{array}{l}\text { 7. Rúbrica de evaluación de } \\
\text { problema de ABP. }\end{array}$ & $\begin{array}{l}\text { Construir y validar la rúbrica de evaluación de los problemas } \\
\text { elaborados por los docentes. }\end{array}$ \\
\hline 8. Reconstrucción del problema. & Reelaborar el problema luego de considerar la evaluación de pares. \\
\hline $\begin{array}{l}\text { 9. Trabajo de ABP con los } \\
\text { estudiantes. }\end{array}$ & $\begin{array}{l}\text { Proponer una secuencia de trabajo con estudiantes que parta de un } \\
\text { problema bajo perspectiva del ABP. }\end{array}$ \\
\hline $\begin{array}{l}\text { 10. Evaluación del ABP y del } \\
\text { proceso formativo. }\end{array}$ & $\begin{array}{l}\text { Valorar los alcances y limitaciones del ABP como estrategia y del } \\
\text { proceso desarrollado con los profesores. }\end{array}$ \\
\hline
\end{tabular}

\section{Resultados}

Los resultados se exponen teniendo en cuenta las etapas señaladas en la metodología. Es importante anotar que, en ambos contextos, se consideraron para el desarrollo de la propuesta los principios epistemológicos, pedagógicos y didácticos del ABP. 


\section{Revisión sistemática de la literatura}

Se hizo una búsqueda, localización y sistematización de información. Para la primera y segunda parte, se usaron bases de datos de acceso libre. Para el proceso de sistematización, se establecieron criterios previos, entre los que se destacan: génesis del $\mathrm{ABP}$, marco epistemológico, características, experiencias de aula y trabajos de investigación. Luego se hizo una lectura crítica de los textos seleccionados, según algunos planteamientos de Garcés y Duque (2007) y de Díaz (2009).

\section{Características del ABP como estrategia}

De manera general, al tener presente que el ABP fomenta la formación integral y holística de los estudiantes, en la tabla 2 se presentan los aspectos que favorecen esta estrategia de aprendizaje por indagación, organizados en relación con la construcción del sujeto que aprende. Dichos aspectos fueron discutidos y establecidos previamente por los autores a partir de la revisión teórica. Sin embargo, se incluyeron unos nuevos, que surgieron de la experiencia de dos años de investigación. Estos son fundamentales para los docentes que quieran implementar la estrategia de ABP.

\section{Tabla 2}

Características del $A B P$

- Destaca el papel activo del sujeto — tanto del que aprende, como del que diseña la estrategia-.

- La construcción de conocimiento se da como un proceso - no en forma puntual

En relación con el y lineal—, que se desarrolla en interacción con los otros y genera conflicto cognitivo en el sujeto que lo construye.

conocimiento

- El profesor es un mediador (Bruner, 2008). El conocimiento se desarrolla mediante el reconocimiento y la aceptación de los procesos sociales y de la evaluación de las diferentes interpretaciones individuales del mismo fenómeno.

- El profesor diseña actividades que orientan el cambio conceptual (Pozo, 2006).

- La pregunta es clave para favorecer la indagación, porque en esta estrategia no se trata de dar respuestas, sino de elaborar preguntas que ayuden a desarrollar el pensamiento (Short et al., 1999).

- El profesor es un aprendiz. Es decir, no debe ser necesariamente un experto sobre el tema o sobre los aspectos del problema.

En relación con la enseñanza

- Los profesores diseñan problemas abiertos, para ser resueltos por los estudiantes; y no el contenido en exposiciones de acuerdo con la disciplina.

- Generalmente el profesor establece una secuencia conceptual y temática estática. En el ABP, es más flexible, abierta y dinámica.

- Los conceptos, tópicos y temas se abordan desde unas necesidades que son esenciales para resolver un problema y, por ello, cobran mayor sentido en el estudiante.

- Se centra en el alumno y el desarrollo de sus habilidades y competencias - y no en los contenidos descontextualizados y fragmentados-, lo cual favorece el aprendizaje significativo (Pérez et al., 2007).

- Se promueve la aprendizaje situado y contextualizado.

- Potencia el pensamiento crítico. Para ello, es necesario generar escenarios o

En relación con el aprendizaje situaciones prácticas, como los problemas, ya que este no se puede desarrollar en abstracto (Kabalem, 2012; Quintero et al., 2017; Sola, 2006a, 2006b ).

- Desarrolla competencias en contextos y situaciones específicas al hacer uso de procesos cognitivos de orden superior a partir de la trasferencia y la creación de soluciones.

- Le apunta al desarrollo de habilidades cognitivas de nivel superior como análisis, síntesis e innovación.

- Motiva la investigación, la indagación y el pensamiento científico.

- Favorece el reconocimiento de las capacidades diversas de los estudiantes. 
- Estimula el trabajo cooperativo y permite que los estudiantes asuman diferentes roles y construyan interdependencia positiva de manera continua y sistemática (Johnson et al.,1991).

En relación con el trabajo en equipo

- Fomenta actitudes y valores de respeto y tolerancia.

- Promueve la responsabilidad y acciones individuales y colectivas importantes en el proceso de formación de todo individuo (Poot-Delgado, 2013).

\begin{tabular}{ll}
\hline $\begin{array}{l}\text { En relación con la } \\
\text { construcción de } \\
\text { identidad sobre } \\
\text { quien escribe y } \\
\text { habla }\end{array}$ & $\begin{array}{l}\text { - Abre espacios para reconocer las fortalezas y debilidades. } \\
\text { Favorece la construcción de una voz propia que se refleja en la escritura } \\
\text { académica, en las discusiones y en debates entre pares y con expertos. }\end{array}$ \\
\hline $\begin{array}{l}\text { En relación con } \\
\text { los contextos } \\
\text { históricos y } \\
\text { geográficos }\end{array}$ & $\begin{array}{l}\text { - Aporta soluciones a problemas locales, regionales y nacionales. } \\
\text { En relación con }\end{array}$ \\
$\begin{array}{l}\text { la regulación } \\
\text { y procesos } \\
\text { metacognitivos }\end{array}$ & $\begin{array}{l}\text { - Desarrolla autonomía y procesos reflexivos continuos para reconocer la validez de } \\
\text { las hipótesis y de las soluciones presentadas a los problemas abordados. }\end{array}$ \\
\hline $\begin{array}{l}\text { Promueve en el estudiante la responsabilidad de su propio proceso de aprendizaje. } \\
\text { En relación con la } \\
\text { evaluación }\end{array}$ & $\begin{array}{l}\text { - Se establecen los criterios con anterioridad, los cuales son conocidos por el grupo } \\
\text { de estudiantes. }\end{array}$ \\
\hline $\begin{array}{l}\text { La evaluación es continua, holística e integral (López, 2008). } \\
\text { Se puede monitorear y hacer seguimiento a partir de rúbricas de evaluación. }\end{array}$ \\
\hline
\end{tabular}

\section{Planeación y diseño de la estrategia con los docentes en formación y en ejercicio}

En esta etapa, los autores definieron los objetivos, los recursos, las lecturas y los productos esperados en cada una de las sesiones, entre otros aspectos. Para el caso de los estudiantes de la licenciatura, la estrategia se organizó en función de las 16 semanas del semestre. Para los docentes en ejercicio, se establecieron encuentros semanales a lo largo del año, de tal manera que se lograra no solo identificar la estrategia, sino comprenderla, analizarla y adecuarla a los diferentes grados. El diálogo entre la teoría y la práctica permitió enriquecer la propuesta de cada equipo de trabajo y la reflexión en y sobre la acción que, además, posicionó a los maestros desde el lugar de profesionales reflexivos de sus prácticas (Schön, 2002).

\section{Etapa de sensibilización}

Los verdaderos cambios en la escuela no surgen por modas pedagógicas, por dar nuevas denominaciones a viejas prácticas o por consumir acríticamente teorías. Las propuestas de innovación tienen que ver, sobre todo, con la manera como la institución educativa y sus agentes construyen escenarios creativos en los que se potencien los aprendizajes de la comunidad, en los que se observen y se analicen los contextos, desde la construcción de un acervo teórico que sustente y fundamente las decisiones y acciones del docente. Pero estos aprendizajes están marcados por una apuesta fundamental: el deseo. En ese sentido, el deseo se convierte en el pivote que proyecta un nuevo escenario, en el motor que moviliza las acciones de los maestros y de la institución, pues es desde el lugar de cada uno de los sujetos que se enuncia, de manera activa y dinámica, la construcción de conocimientos que generan nuevas apuestas para la educación. Ese es el reto de una institución educativa: construir conjuntamente el espacio de cambio, en el que se acerque más la teoría a la práctica. Para lograrlo, es necesario observar cómo las diferentes investigaciones de las ciencias cognitivas, 
las teorías pedagógicas y las didácticas permiten comprender mejor el aprendizaje, pero también el lugar de los profesores y de los estudiantes en este escenario educativo.

\section{Fundamentación teórica}

Por las razones expuestas en el apartado anterior, la teoría cobra sentido, al abrir espacios que promuevan la participación de todos los docentes y el trabajo en equipo, al discutir críticamente los textos de la literatura que circulan e, igualmente, al construir las unidades y/o estrategias didácticas, su forma de desarrollo y evaluación. Estas acciones permiten construir comunidad de práctica y de aprendizaje (Wenger, 2000), en donde la voz de los docentes tiene una identidad en los textos que elaboran para los estudiantes y en las actividades que proponen en el aula, al tiempo que se convierten en un espacio de debate y preguntas.

Esta apuesta exige una construcción polifónica que rompa las barreras de los planes en solitario: abrir nuestras aulas. Implica también compartir con los colegas espacios para compartir nuestras emociones (Henao-Arias et al., 2017), saberes, y reconocernos en un espacio creativo en donde las cogniciones se conectan al ampliar saberes y consolidar otros, pero también con la construcción de nuevas soluciones para enriquecer el aula y las prácticas pedagógicas. De la lectura crítica que desarrollaron los docentes en formación y en ejercicio, se identificaron tópicos que fueron objeto de estudio y de reflexión en términos de las ventajas del ABP, el aprendizaje y los factores asociados.

\section{Diseño del problema}

Se partió de discutir colectivamente el concepto de problema, especialmente en el marco de la estrategia de ABP. Se retomaron las definiciones de Krulik y Rudnik (1980) y de Polya (1980): los primeros establecen que un problema es una situación, que puede ser cualitativa o cuantitativa, mientras que quienes pretenden solucionarla no cuentan con los recursos y caminos para hacerlo; en la segunda se señala que en la solución de un problema son necesarias dos cosas: (1) encontrar una ruta nueva, la cual no se vislumbra de manera inmediata; y (2) vencer las dificultades que se puedan presentar en el camino, es decir, en el objetivo de solucionar el problema identificado. En síntesis, en la definición que se construye con los profesores, se considera que "un problema es una situación de carácter local, nacional o internacional, que plantea interrogantes y despierta interés y motivación de soluciones novedosas por parte de los estudiantes". Para el diseño del problema se tuvo en cuenta que:

a. Fuera auténtico y estuviera basado en una situación real.

b. Fuera cercano al estudiante, o de interés global, como el coronavirus, por ejemplo.

c. Describiera la actualidad local, nacional o internacional.

d. En su estructura no incluyera todas las piezas.

e. No tuviera una única solución.

f. Abriera posibilidades a la imaginación, la creatividad y la innovación.

g. Lograra conectar con los conocimientos previos de los estudiantes.

h. Generara controversia.

i. Despertara el interés de los estudiantes y motivara a su solución (Jonassen, 2000; Talbert-Johnson \& Oberlander, 2004).

j. Valoraran la complejidad del problema. Esta característica está dada por el nivel de escolaridad y el desarrollo psicológico e intelectual de los estudiantes.

k. Variara de un grupo a otro, en función de sus necesidades. 
Es fundamental que los profesores tengan siempre presentes en el diseño del problema las competencias, las habilidades y los contenidos que deben ser potenciados y reconstruidos. Se sugiere que el diseño se haga en equipos de docentes, preferiblemente de áreas de conocimiento diferentes, que prevean los alcances y las posibles limitaciones que exige el mismo. El problema se estructura en un texto, generalmente de carácter informativo y/o expositivo, que considera, entre otras cosas, el lenguaje, la redacción, las imágenes, las tablas y las fotografías, entre otros recursos; y, por supuesto, las preguntas. Estas deben cumplir con ciertas características: estar bien formuladas, ser abiertas, que no tengan una única respuesta, que favorezcan el desarrollo de las competencias, que tengan en cuenta no solo aspectos de orden conceptual, sino también procedimental, actitudinal y valorativo, que estén vinculadas y sean detonantes de los conocimientos previos e, igualmente, que estimulen la creatividad e innovación de los estudiantes.

\section{Construcción de rúbrica de evaluación de problema de $A B P$}

Una rúbrica es un registro que contempla criterios a evaluar. Se diseña teniendo en cuenta procesos intelectuales, actitudinales, axiológicos y comunicativos, según unos niveles que dan cuenta del desarrollo cognitivo, que sirven para situar los niveles de apropiación conceptual. Permite desarrollar conciencia sobre la tarea, al posibilitar a los estudiantes y maestros reconocer las expectativas compartidas sobre el aprendizaje (Torres \& Perera, 2010). En esta etapa, los investigadores, reconocieron la importancia de la construcción compartida de las rúbricas. Producto de este trabajo, se construyó el instrumento que se presenta en la tabla 3:

Tabla 3

Rúbrica de evaluación del diseño del problema

\begin{tabular}{|c|c|c|c|c|}
\hline \multirow[t]{2}{*}{ Categoría } & \multirow[t]{2}{*}{ Aspectos importantes a considerar } & \multicolumn{2}{|c|}{$\begin{array}{c}\text { Escala } \\
\text { de valoración }\end{array}$} & \multirow[t]{2}{*}{ Observaciones } \\
\hline & & Sí & No & \\
\hline \multirow{10}{*}{$\begin{array}{l}\text { Aspecto } \\
\text { conceptual }\end{array}$} & $\begin{array}{l}\text { ¿En el problema se identifican los conceptos } \\
\text { sobre los contenidos objeto de estudio? }\end{array}$ & & & \\
\hline & $\begin{array}{l}\text { ¿El problema favorece el desarrollo de las } \\
\text { habilidades y competencias establecidas en el } \\
\text { plan de estudios? }\end{array}$ & & & \\
\hline & $\begin{array}{l}\text { ¿En el problema se identifica claramente la } \\
\text { integración de las áreas? }\end{array}$ & & & \\
\hline & ¿El problema fomenta el análisis y la predicción? & & & \\
\hline & $\begin{array}{l}\text { ¿Los contenidos conceptuales temáticos del } \\
\text { problema son pertinentes para el nivel de la } \\
\text { complejidad con el curso y la edad? }\end{array}$ & & & \\
\hline & $\begin{array}{l}\text { ¿Los datos del problema, cualitativos y/o } \\
\text { cuantitativos, son los necesarios para comprender } \\
\text { el problema y ubicar su contexto? }\end{array}$ & & & \\
\hline & $\begin{array}{l}\text { ¿El problema permite plantear múltiples } \\
\text { soluciones? }\end{array}$ & & & \\
\hline & ¿El problema genera reto cognitivo? & & & \\
\hline & ¿El problema favorece el pensamiento crítico? & & & \\
\hline & $\begin{array}{l}\text { ¿El problema favorece la construcción de } \\
\text { diferentes tipos de conocimiento? }\end{array}$ & & & \\
\hline
\end{tabular}




\begin{tabular}{|c|c|}
\hline \multirow{5}{*}{$\begin{array}{c}\text { Aspecto } \\
\text { procedimental }\end{array}$} & $\begin{array}{l}\text { ¿El problema aproxima a los estudiantes a } \\
\text { comprender cómo se genera el conocimiento } \\
\text { científico? }\end{array}$ \\
\hline & ¿El problema activa conocimientos previos? \\
\hline & $\begin{array}{l}\text { ¿Los estudiantes poseen el suficiente y relevante } \\
\text { conocimiento previo para afrontar el problema? }\end{array}$ \\
\hline & $\begin{array}{l}\text { ¿El problema estimula la búsqueda de información } \\
\text { y el uso de fuentes de consulta? }\end{array}$ \\
\hline & $\begin{array}{l}\text { ¿El problema está bien estructurado en relación } \\
\text { con el contexto local, nacional e internacional? }\end{array}$ \\
\hline \multirow{2}{*}{$\begin{array}{l}\text { Aspectos } \\
\text { afectivos y } \\
\text { valorativos }\end{array}$} & $\begin{array}{l}\text { ¿El problema puede provocar interés y motivación } \\
\text { en la búsqueda de su solución? }\end{array}$ \\
\hline & $\begin{array}{l}\text { ¿El problema puede promover el análisis reflexivo } \\
\text { en relación con las diversas formas de pensar, } \\
\text { sentir y actuar? }\end{array}$ \\
\hline \multirow{3}{*}{$\begin{array}{l}\text { Aspectos } \\
\text { relacionados } \\
\text { con las } \\
\text { preguntas }\end{array}$} & ¿Las preguntas están bien formuladas? \\
\hline & $\begin{array}{l}\text { ¿Las preguntas potencian el desarrollo de las } \\
\text { habilidades y competencias? }\end{array}$ \\
\hline & $\begin{array}{l}\text { ¿Las preguntas se organizan en orden de } \\
\text { complejidad? }\end{array}$ \\
\hline \multirow{7}{*}{$\begin{array}{l}\text { Aspectos } \\
\text { relacionados } \\
\text { con el texto }\end{array}$} & ¿El texto cumple con el propósito comunicativo? \\
\hline & ¿El texto está bien redactado? \\
\hline & $\begin{array}{l}\text { ¿El texto expositivo presenta secuencias de } \\
\text { analogías y ejemplos? }\end{array}$ \\
\hline & $\begin{array}{l}\text { ¿En el texto se enuncia el problema de manera } \\
\text { explícita? }\end{array}$ \\
\hline & $\begin{array}{l}\text { ¿El contenido de los recursos gráficos - imágenes, } \\
\text { infografías, fotos y tablas, entre otros- está } \\
\text { relacionado con el problema planteado? }\end{array}$ \\
\hline & $\begin{array}{l}\text { ¿En el texto se mencionan las fuentes de consulta } \\
\text { para plantear el problema? }\end{array}$ \\
\hline & ¿Los recursos gráficos usados tienen fuente? \\
\hline
\end{tabular}

\section{Reconstrucción del problema}

En esta etapa, se generaron diferentes momentos para la lectura y el análisis de los problemas desarrollados por los equipos de trabajo. La posibilidad de ser leídos y de discutir con colegas de diferentes niveles permitió construir un clima que abre las puertas del aula, pues de lo que se trataba era de poner en común una apuesta didáctica construida de manera cooperativa, al dejar de lado los currículos privados y cerrados. Otro aspecto valioso estuvo relacionado con la manera como se recogieron y se asumieron las observaciones, pues se reconocieron los saberes y experticias de los otros, como interlocutores válidos. Los tres agentes de la evaluación - heteroevaluación, coevaluación y autoevaluación - se vivieron en esta etapa, que les permitió a los docentes desarrollar estrategias metacognitivas sobre el proceso.

\section{Trabajo de ABP con los estudiantes}

Teniendo en cuenta la premisa inicial, los profesores en formación y en ejercicio reflexionaron sobre algunos aspectos a la hora de abordar el problema con los estudiantes. A continuación, de manera sucinta, se describen estos, que fueron recogidos por los autores en los diferentes grupos con los que se desarrolló la experiencia. 
Presentación del problema. El problema se puede presentar de diversas maneras. Depende de la creatividad de los maestros que lo diseñan. Puede ser en fotocopia, video, correo electrónico, WhatsApp, página web, emisora del colegio, Facebook o en una pequeña pieza de teatro que ambiente la situación a manera detectivesca, entre otras. Lo más importante es que, sin importar el medio de presentación, se sugiere leer y analizar el problema con los estudiantes. Esta actividad es clave para la activación de conocimientos previos, para que los estudiantes identifiquen y caractericen el escenario y contenido del problema, que tiene que ver con el espacio geográfico, el tiempo, los actores que están involucrados, los conceptos, los temas y el tipo de relaciones que estos establecen entre sí.

Trabajo cooperativo. Es fundamental propiciar discusiones y grupos cooperativos que permitan trabajo desde roles específicos, al reconocer:

- Interdependencia positiva. Cada uno puede mostrar lo aprendido por separado y ser valorado por ello, pero también mostrar lo que aprenden juntos - que fue el énfasis del taller-.

- La vida en equipo. Hay que reconocer que el aprendizaje depende de todos, que es de largo aliento y que conlleva la participación en todas las actividades.

Estos aspectos fueron considerados no solo para la implementación del ABP con los estudiantes, sino en el proceso mismo de formación docente.

Construcción de la hipótesis. Los estudiantes plantean soluciones iniciales. Es importante que el docente aproveche este espacio para compartir los supuestos de cada grupo. Las primeras hipótesis le sirven al docente para identificar conocimientos previos, vacíos conceptuales, tipo de conocimientos y experiencias. Estas evidencias posibilitan la construcción de andamiajes para trabajar sobre los objetivos de aprendizaje y los niveles de desarrollo de cada estudiante. Desde la misma presentación del problema, es necesario orientar a los estudiantes en el reconocimiento de los objetivos de aprendizaje y lo que se espera que ellos realicen - aspecto necesario para construir un esquema de trabajo-.

Cada grupo debe diseñar su plan de acción. Para ello, se requiere, entre otras cosas, identificar los conceptos y procesos que les son familiares, sobre los que tienen un dominio y apropiación, como también aquellos que les son desconocidos, que son fundamentales y que se necesitan para comprender en su totalidad la magnitud y los alcances del problema, como también para esbozar posibles soluciones, que se pueden representar en un organizador gráfico. Es importante anotar que la revisión del problema se debe ir haciendo en la medida que avanza tanto la consulta como la información que se recaba.

Consulta de información y validación de hipótesis. En esta fase, los estudiantes consultan libros, revistas, enciclopedias, bases de datos y expertos. Las visitas pedagógicas a museos, empresas, sitios históricos, entre otros, son también fuente de información. Se pueden sugerir y organizar talleres, conferencias y laboratorios. La propuesta apunta a que los estudiantes aprendan a indagar, localizar y usar los buscadores, así como a seleccionar la información —el manejo de fuentes confiables y distinguir las que no lo son-. Es necesario trabajar en el uso y referenciación de fuentes.

Planteamiento de la solución y validación de hipótesis. Los estudiantes, después de analizar todas las fuentes posibles, proponen soluciones a los problemas, que deben ser 
justificadas y razonadas. Los juicios de valor que se desprendan de dichas decisiones deben estar basados en información, hechos, evidencias y posibilidades de desarrollo. Se requiere lectura crítica de toda la información recabada con respecto al problema, ya que favorece, entre otras cosas, la selección de las fuentes con información relevante, los procedimientos y los pasos que se deben desarrollar para solucionar el problema. La validación de hipótesis permite, desde la metacognición, evaluar el proceso mismo de aprendizaje, en la medida que reconoce los grados de elaboración conceptual, la manera como se reconstruye el conocimiento, los conflictos cognitivos para resolver la situación presentada, la apropiación de nuevos conceptos y la contrastación con otras posibles soluciones que plantean los compañeros.

Socialización y realimentación. Los hallazgos se deben compartir en la clase. Este espacio se aprovecha para analizar la argumentación, las fortalezas y debilidades de cada grupo. La realimentación, básica para el aprendizaje, es continua y se da durante todo el proceso. Se recomienda que se abran espacios formales con esta intención, de modo que sirvan para reconocer avances y aspectos por mejorar. También pueden ser aprovechados para motivar y repensar las soluciones a los problemas, en caso de ser necesario. Fue importante, en esta etapa, la forma como los estudiantes aprendieron a reconocer las carencias y fallas, como también a valorar el trabajo individual y colectivo.

La evaluación. Se debe entender la evaluación como un proceso continuo que se va dando a lo largo de la situación de aprendizaje diseñada en el ABP en cada una de las etapas. Es integral, en la medida que considera las competencias, habilidades y los contenidos, así como los aspectos axiológicos de trabajo en equipo - respeto, escucha activa, negociación de acuerdos y significados-. Además, permitió establecer niveles de avance. La forma como los estudiantes participaron de su propio proceso de aprendizaje a través de la autoevaluación y de la coevaluación fue importante para el cambio conceptual. A continuación, se describen algunos aspectos que fueron objeto de evaluación en los estudiantes de manera individual y grupal.

- Preparación de las sesiones.

- Participación y contribución al trabajo del grupo.

- Responsabilidad con los compromisos individuales y grupales.

- Habilidades intrapersonales e interpersonales.

- Motivación interna y externa.

- Reconocimiento de los aportes de los compañeros.

- Sentido de tolerancia y respeto frente a las fallas, a errores o, incluso, a los defectos de los compañeros.

En relación con los tipos de evaluación, se construyeron instrumentos variados y se consideraron sus potencialidades y limitaciones. Se presentan a continuación algunos:

- Organizadores gráficos: ayudan a representar conocimientos y relaciones lógicas.

- Reportes escritos: informes de avances.

- Exposiciones orales: habilidades comunicativas y oralidad formal.

- Trabajos prácticos: evidencian aspectos de orden procedimental. Práctica de laboratorio.

- Exámenes: preguntas abiertas con libro abierto donde tengan que analizar datos, graficarlos, sacar conclusiones, proponer soluciones y aplicar conceptos a nuevas 
situaciones, entre otros.

- Pruebas que sigan los modelos censales, nacionales e internacionales -PISA, TIMMS, SERCE, Pruebas Saber-.

- Diarios de aprendizaje: reflexiones de carácter individual que hace el estudiante con respecto sus responsabilidades, avances, dificultades y por supuesto aprendizajes construidos.

- Portafolios: permite recolectar las evidencias de aprendizaje de los estudiantes. A su vez, posibilita la reflexión sobre el desarrollo, ya que se monitorean sus avances y se reconocen los niveles de las competencias alcanzadas.

Evaluación del ABP y del proceso formativo. En esta etapa se consideraron dos aspectos: por un lado, la evaluación que hacen los docentes que participaron; y, por otro, la que hacen los autores en relación con la estrategia de formación y el proceso desarrollado. En cuanto a la primera parte, se destaca cómo esta estrategia les permitió vincularse más con sus colegas, al reconocer nuevas dinámicas de trabajo y al realizar desde allí una apuesta mucho más integrada y articulada, lo que propició la integración de saberes disciplinares para apuntar a un currículo integrado (Torres, 2015). Por otra parte, se reconoció cómo las prácticas de lectura y escritura, que actualmente se desarrollan a través de las TIC, se vincularon en toda la experiencia, pues los docentes se convirtieron en lectores y escritores en las diferentes fases y reconocieron la función epistémica de la escritura, como un instrumento fundamental para desarrollar el pensamiento.

Así mismo, la reflexión que hicieron los maestros, tanto en formación como en ejercicio, sobre qué es lo fundamental que los estudiantes aprendan desde el ámbito STEM les permitió reconocer el valor de los conceptos estructurantes y de los procesos de transposición didáctica, en tanto privilegiaron la profundidad de los conceptos a la extensión de contenidos. Reconocieron también la necesidad de aproximarse a nuevas formas de explorar los procesos de enseñanza y aprendizaje y reflexionar sobre ellos.

Igualmente, abrir un espacio de diseño y planeación conjunta, así como de clases compartidas, enriqueció no solo a los estudiantes, sino a los mismos maestros, pues este ejercicio dialógico de intercambios posibilitó varias miradas tanto en el diseño como en el proceso de acompañamiento que los maestros realizaron en la solución del problema, ya que integraron saberes y analizaron perspectivas que reconocen, en últimas, que el aprendizaje se da gracias a la posibilidad de encontrar esos vasos comunicantes de las disciplinas y de un currículo integrado.

A partir del proceso metacognitivo que se realizó en cada fase de formación, los maestros reflexionaron sobre los aprendizajes logrados como los que arriba se señalaron, así como las limitaciones de la estrategia asociadas con el tiempo. Por último, los investigadores manifiestan su agradecimiento a los estudiantes y profesores que hicieron parte de este proceso. Sus valiosos aportes y su interés por el trabajo desarrollado permitieron llevar a cabo esta experiencia.

\section{Conclusiones}

La investigación permitió un proceso de cualificación alrededor de una estrategia de aprendizaje por indagación como el ABP, que favoreció aprendizajes tanto para los docentes 
como para los estudiantes y, a su vez, movilizó los currículos de la institución. En primer lugar, los docentes reconocieron la potencia de trabajar de manera interdisciplinaria en el diseño, el desarrollo y la implementación del ABP. Abrieron sus puertas del aula al trabajo cooperativo y lograron aprender y reflexionar sobre procesos didácticos e investigativos para favorecer los aprendizajes. Por otro lado, llevar al aula problemas reales como la polución, la contaminación, los recursos hídricos, la extinción de las abejas y el coronavirus, entre otros, se convirtió en el motor para el desarrollo de hipótesis y su confrontación, a partir de un proceso constante de investigación cooperativa y de actividades que ponen en el centro al estudiante, quien reconoce la potencia del diseño y la mediación del maestro.

Los maestros, al apropiarse de la estrategia, reconocieron que, aunque al principio fue difícil el trabajo cooperativo, tanto la evaluación como las clases fueron más enriquecidas por los debates y aportes de los colegas. Se amplió la mirada de la evaluación, de la investigación y del pensamiento, pues de lo que se trató fue de construir debates y argumentaciones sobre las soluciones planteadas y su puesta en marcha. Reconocieron, en varios casos, cómo los estudiantes ejercieron sus derechos ciudadanos, al escribir cartas a los alcaldes de municipios colombianos. Así mismo, generaron estrategias de cambio, al proponer soluciones a las inundaciones, el diseño de jardines para la polinización, el uso y el diseño de paneles solares, entre otros.

En relación con los estudiantes, el aprendizaje activo fue el aspecto que más reconocieron. La motivación de sentirse parte de la solución fue fundamental. El hecho de plantear hipótesis que eran constantemente reformuladas les permitió reconocer el error como fuente de aprendizaje, pero además les ayudó a consolidar el pensamiento argumentativo y crítico. Incluso al final, ellos mismos llevaron al aula otros problemas que consideraban importantes para trabajar. Para los investigadores, el proceso de dos años de trabajo permitió reconocer la importancia del acompañamiento constante en los procesos de formación docente desde la reflexión de las actividades en comunidades de práctica y aprendizaje que se vinculan desde el deseo de cambio.

\section{Referencias}

Andreu-Andrés, M., \& García-Casas, M. (2006). Evaluación, coevaluación y autoevaluación del trabajo en grupo en la lectura de mapas topográfico. En F. Watts \& A. GarcíaCarbonell (Eds.), La evaluación compartida: investigación multidisciplinar (pp. 69-90). Universidad Politécnica de Valencia.

Bruner, J. (2008). Acción, pensamiento y lenguaje. Alianza Editorial.

Cohen-Schotanus, J., Muijtjens, A., Schonrock-Adema, J., Geertsma, J., \& Vleuten van der, C. (2008). Effects of conventional and problem-based learning on clinical and general competencies and career development. Medical Education, 42(3), 256-265. https://doi. org/10.1111/j.1365-2923.2007.02959.x

Coll, C. (1998). La teoría genética y los procesos de construcción del conocimiento en el aula. En J. Castorina, C. Coll A. Diáz, F. Díaz, B. Areco; G. García; L. Hernández; L. Moreno, A. Muriá, \& C. Pessoa (Eds.), Piaget en la educación. Debate en torno a sus aplicaciones (pp. 15-52). Paidós, UNAM. 
Díaz, J. (2009). Guía práctica de lectura crítica de artículos científicos originales en ciencias de la salud. Instituto Nacional de Gestión Sanitaria. https://www.sepeap.org/wp-content/ uploads/2015/06/Guia_practica_de_lectura.pdf

Garcés, J., \& Duque, J. (2007). Metodología para el análisis y la revisión crítica de artículos de investigación. Innovar, 17(9), 184-194. http://www.scielo.org.co/pdf/inno/v17n29/ v17n29a11.pdf

Henao-Arias, J., Vanegas-García, J., \& Marín-Rodríguez, A. (2017). La enseñanza en vilo de las emociones: una perspectiva emocional de la educación. Educación y Educadores, (3), 451-465. https://doiorg/10.5294/edu.2017.20.3.7

Johnson, D., Johnson, R., \& Smith, K. (1991). Active learning: cooperation in the college classroom. Interaction Book Company.

Jonassen, D. (2000). El diseño de entornos constructivistas de aprendizaje. En C. Reigeluth, (Ed.), Diseño de la instrucción. Teoría y modelos (pp. 225-250)Aula XXI Santillana.

Kabalen, D. (2012). Análisis y pensamiento crítico para la expresión verbal. Editorial Digital Tecnológico de Monterrey. https://www.editorialdigitaltec.com/materialadicional/ ID371_

Krulik, S., \& Rudnik, J. (1980). Problem solving in school mathematics. National Council of Teachers of Mathematics.

López, M. (2008). El aprendizaje basado en problemas. Una propuesta en el contexto de la educación superior en México. Tiempo de Educar, 9(18), 199-232. https://www.redalyc. org/pdf/311/31111811003.pdf

Oakley, B., Hanna, D., Kuzmyn, K., \& Felder, R. (2007). Best practices involving teamwork in the classroom: Results from a survey of 6435 engineering student respondents. IEEE Transactions on Education, (50), 266-272. http://www4.ncsu.edu/unity/lockers/users/f/ felder/public/Papers/OakleyPaper(IEEE).

Pérez, J., Baños, J., Calafell, F., Carrió, M., \& Larramona, M. (2007) Introducción del aprendizaje basado en problemas en la titulación de Biología de la Universidad Pompeu Fabra. En Centro de Innovación y Documentación Educativa. Premios Nacionales de Innovación Educativa 2006 (pp. 93-111). Ministerio de Educación y Ciencia.

Polya, G. (1980). Cómo plantear y resolver problemas. Trillas.

Poot-Delgado, C. (2013). Retos del aprendizaje basado en problemas. Enseñanza e Investigación en Psicología, 18(2), 307-314. https://www.redalyc.org/pdf/292/29228336007.pdf

Pozo, J. (2006). La nueva cultura del aprendizaje en la sociedad del conocimiento. En J. Pozo, N. Scheuer, M. Pérez, M. Mateos, E. Martín, \& M. de la Cruz (Eds.), Nuevas formas de pensar la enseñanza y el aprendizaje: las concepciones de profesores y alumnos (pp. 2954). Graó.

Prince, K., Eijs van, P., Boshuizen, H., Vleuten van der, C., \& Scherpbier, A. (2005). General competencies of problem-based learning (PBL) and non-PBL graduates. Medical Education, 39(4), 394-401. https://doi.org/10.1111/j.1365-2929.2005.02107.x 
Quintero, L., Ávila, J., \& Olivares S. (2017). Desarrollo del pensamiento crítico mediante la aplicación del Aprendizaje Basado en Problemas. Psicología Escolar e Educacional. 21(1), 65-77. https://doi.org/10.1590/2175-3539201702111072

Rhem, J. (1998). Problem-based learning: An introduction. The National Teaching and Learning Forum, 8(1), 1-4.

Schön, D. (2002). La formación de profesionales reflexivos. Hacia un nuevo diseño de la enseñanza y el aprendizaje en las profesiones. Paidós.

Short, K., Choroeder J., Laird, J., Kauffman, G., \& Ferguson. (1999). El aprendizaje a través de la indagación. Gedisa Editorial.

Sola, C. (2006a). Fundamentos de la técnica didáctica ABP. En M. Porres, C. Sola, R. Gentil, L. Epstein, G. Lapuente, S. Limón, F. Sierra, L. Neri, M. McCoy, J. Álvarez, L. Delgado, \& F. Illescas (Eds.), Aprendizaje basado en problemas: de la teoría a la práctica (pp. 37-50). Trillas.

Sola, C. (2006b). Ventajas y desventajas de ABP: A modo de final. En M. Porres, C. Sola, R. Gentil, L. Epstein, G. Lapuente, S. Limón, F. Sierra, L. Neri, M. McCoy, J. Álvarez, L. Delgado, \& F. Illescas (Eds.), Aprendizaje basado en problemas: de la teoría a la práctica (pp. 188-201). Trillas.

Talbert-Johnson, C., \& Oberlander, J. (2004). Teachers in the Trenches: Teacher Education, Technology Integration and the Creation of Technology-Enhanced Environments. Teacher Education and Practice, 17(1), 29-44. https://www.learntechlib. org/p/71516/.

Torres, J.(2015). Sin muros en las aulas. El currículo integrado. En Ensayos sobre el currículo: teoría y práctica. J. Gimeno Sacristán; M, Santos Guerra; J. Torres Santomé, J. Marrero: P. Jackson. (148-169) Editorial Morata.

Torres, J., \& Perera, V. (2010). La rúbrica como instrumento pedagógico para la tutorización y evaluación de los aprendizajes en el foro online en educación superior. Revista de Medios y Educación, (36), 141-149. https://www.redalyc.org/pdf/368/36815128011.pdf

Vygotski, L. (1993). Pensamiento y lenguaje. En A. Zaporozhéts (Comp.), Obras Escogidas. Tomo 2 (pp. 9-348). Visor.

Wenger, E. (2000). Communities of Practice: Learning, Meaning and Identity. Cambridge University Press 